\title{
Histopathological examination of products of conception after first-trimester miscarriage
}

\author{
Thirukumar $\mathbf{M}^{1}$ \\ ${ }^{1}$ Faculty of Health Care Science, Eastern University, Sri Lanka
}

\author{
Article Information \\ Total number of \\ Words 1076 \\ Tables 03 \\ Author has no conflicts of interest to declare \\ Keywords: Retained products of conception, \\ miscarriage, Ultrasound, Histopathological \\ examination
}

Date of submission: 02.12.2018

Date of acceptance: 28.06.2019

Author responsible for correspondence:

Dr. M. Thirukumar

Senior lecturer in Obstetrics and

Gynaecology, Department of Clinical

Sciences

Faculty of Health Care Science, Eastern

University, Sri Lanka

Email: dr.thiru10@yahoo.com

https://orcid.org/0000-0001-8499-7175

DOI: http://doi.org/10.4038/cjms.v56i1.4946

\begin{abstract}
Introduction

This study is aimed to determine the value of histopathological examination of tissues obtained from evacuation of retained products of conception (ERPC) procedure. In addition, it is also aimed to determine the correlation between ultrasonically suspected Hydaditiform mole ( $\mathrm{H}$ Mole) with histopathologically proven $\mathrm{H}$ Mole.
\end{abstract}

\section{Methodology}

In this retrospective study, clinical records of 140 patients who underwent surgical management for early pregnancy loss, from January 2012 to June 2017 were analysed. Their histopathology reports and relevant clinical profiles were collected from medical records.

\section{Results}

The age distribution of studied population ranges from 14 to 52 years. The majority of patients (43.6\%) were within 21-30 years. The Molar pregnancy was confirmed in $32.8 \%$ samples and the majority $(78.3 \%)$ were dominated by complete mole. There were 66 patients ultrasonically suspected to have H Mole. Among them 46 patients had either complete or partial mole. Further, this study showed no molar pregnancies were identified from specimens obtained following evacuation of ultrasonically diagnosed missed or incomplete miscarriage where no $\mathrm{H}$ Mole was suspected.

\section{Conclusion}

It can be recommended that histopathological examination should be performed in selected instances. Further, it is a hospital-based study and its findings increase the importance of a country wide study to guide the revision of current practice of routine histopathological examination of specimens from miscarriages in this middle-income country.

\section{Introduction}

The World Health Organization defines miscarriage either as termination of pregnancy before 20-week gestation or delivery of a foetus weighing less than 500g[1], In Sri Lanka, A loss of pregnancy before 28 weeks of gestation is considered as miscarriage. Miscarriage is one of the most common first-trimester conditions encountered in clinical practice by Obstetricians and Gynaecologists. Approximately 10-20\% of clinical pregnancies are lost spontaneously during first trimester[2]. 
It was believed that retained product of conception (RPOC) increases the risks of infection and haemorrhage and would not be passed spontaneously. Therefore, most women $(88 \%)$ were managed surgically by evacuation of retained products of conception (ERPC), when they had a miscarriage. However, in recent years, management options are widened to include conservative and medical treatment when a woman has a miscarriage[2,3].

Ultrasound is typically the first-line investigation in suspected cases of RPOC. Variable amount of echogenic or heterogeneous material within the endometrial cavity, presence of vascularity within the echogenic material supports the diagnosis and helps to differentiate it from intrauterine blood clot[4].

In most centres, RPOC, obtained by uterine evacuation, is routinely submitted for histopathology examination to confirm the presence of intrauterine foetal tissue or to detect a molar pregnancy. Other reasons include determining the cause of recurrent pregnancy loss or detecting unexpected foetal pathology[5].

It is beneficial to undertake the histopathological examination of the uterine products of conception which was passed spontaneously or evacuated medically or surgically, but it is unclear whether this practice is medically justified. In our institution, the RPOC passed spontaneously or removed during surgical or medical evacuation are not routinely subjected for histopathological examination.

This study is aimed to determine whether histopathology examination of tissues obtained from ERPC has any clinical value and also to determine the correlation between ultrasonically suspected Hydatidiform Mole (HM) with histopathologically proven HM.

\section{Materials and methods}

One hundred and forty (140) patients were recruited for this hospital based, retrospective study from a period of January 2012 to June 2017. They had early pregnancy loss and underwent surgical management.

The retained products of conception obtained by surgical evacuation were submitted for histopathological analysis. All patients' histopathology reports and relevant clinical profiles were retrieved from case records. This research was approved by Ethical review committee of the Faculty of Health Care Science,
Eastern University, Sri Lanka. (EUSL/FHCS/ERC/2017/21).

Data were processed using SPSS version 21. Descriptive statistics methods were used to analyse the results as whole numbers, percentages, tables and charts.

\section{Results}

During the study period, 140 specimens of RPOC were sent for histopathological analysis. The age distribution of patients ranged from 14 to 52 years. The majority of them $(43.6 \%)$ were within the age group of 21-30 years (

Table 1).

The histopathology records revealed that there were no RPOC were detected in 11 patients, even though they were diagnosed to have RPOC by ultrasound.

Molar pregnancy constituted $32.8 \%$ of total samples $(n=46)$ obtained from ERPC for histopathological analysis. The majority (78.3\%) were complete mole and remaining constituted partial mole. The specimens of product of conception were sent for histopathological study following ERPC when HM was ultrasonically suspected. It constituted 66 samples, among them 46 patients were finally found to have either complete or partial mole. None of the molar pregnancies were identified either from specimens obtained following evacuation of ultrasonically diagnosed missed or incomplete miscarriage or from ultrasonically undiagnosed HM during the study period (Table 3 ).

Table 1. Age distribution of study population

\begin{tabular}{|c|c|c|}
\hline $\begin{array}{c}\text { Age groups } \\
\text { (years) }\end{array}$ & $\begin{array}{c}\text { Frequency } \\
\text { (n) }\end{array}$ & $\begin{array}{c}\text { Percentage } \\
(\boldsymbol{\%})\end{array}$ \\
\hline $14-20$ & 21 & 15.0 \\
$21-30$ & 61 & 43.6 \\
$31-40$ & 41 & 29.3 \\
$41-50$ & 16 & 11.4 \\
$\geq 51$ & 1 & 0.7 \\
Total & $\mathbf{1 4 0}$ & $\mathbf{1 0 0 . 0}$ \\
\hline
\end{tabular}

\section{Discussion and conclusion}

Health et al[5], found only two $(0.13 \%)$ molar 
Table 2. Histopathology of the samples obtained from ERPC

\begin{tabular}{|l|c|c|}
\hline & Frequency & Percentage (\%) \\
\hline Products of conception & 83 & 59.3 \\
No products of conception & 11 & 7.9 \\
Complete hydatidiform mole & 36 & 25.7 \\
Partial hydatidiform mole & 10 & 7.1 \\
Total & $\mathbf{1 4 0}$ & $\mathbf{1 0 0}$ \\
\hline
\end{tabular}

Table 3. Ultrasound (USS) findings of patients who underwent evacuation of retained products of conception (ERPC)

\begin{tabular}{|l|c|c|}
\hline USS findings & Frequency & Percentage (\%) \\
\hline Hydatidiform mole corresponding with histopathology & 43 & 30.7 \\
\hline Hydatidiform mole not corresponding with histopathology & 23 & 16.4 \\
\hline Missed miscarriage & 20 & 14.3 \\
\hline Incomplete miscarriage & 40 & 28.6 \\
\hline Increased endometrial thickness & 13 & 9.3 \\
\hline Septic miscarriage & 1 & 0.7 \\
\hline Total & $\mathbf{1 4 0}$ & $\mathbf{1 0 0}$ \\
\hline
\end{tabular}

pregnancies among 1576 patients on histopathology examination of tissue removed during therapeutic miscarriage or emergency uterine evacuation for a spontaneous miscarriage. These authors recommended histopathology evaluation only for patients with an uncertain preoperative diagnosis or those in whom a small amount of tissue or trophoblastic tissue was identified during ERPC.

Histopathology examination of 670 unselected cases at Charing Cross Hospital of London, showed that $120(18 \%)$ patients with unsuspected molar pregnancy were diagnosed with moles based on abundant trophoblast in early pregnancy or the presence of hydrops[6].

A hydatidiform mole is usually first suspected by ultrasound and later confirmed by histopathology. A retrospective analysis of 90 cases of ultrasonographically suspected and histologically proven HM showed that ultrasonography was more reliable for diagnosing complete molar than partial molar pregnancy. The sensitivity of ultrasound for predicting HM was $44 \%-95 \%$ for complete hydatidiform and only $20 \%$ for partial hydatidiform[7], Similarly, Sebire et al. noted that molar pregnancy was suspected on ultrasound in only $34 \%$ of 155 women with molar pregnancies confirmed by histopathology[8], The study by Sharifa Ali Alsibiani advice selective samples, instead of universal, should be sent for histopathological examination[9].

A study, by Oliver $\mathrm{M}$ et al, found that 13 patients with ultrasound scan diagnosis of molar pregnancy who underwent suction curettage, only $9(69.2 \%)$ were confirmed to be complete moles[10].

This current study showed strong correlation between ultrasonic suspicions of HM and histopathological diagnosis of HM. The ultrasound showed a sensitivity of $69.7 \%$ and specificity of $100 \%$ in detecting HM. Based on this finding, the cost of histopathological examinations and the low incidence of molar pregnancies, it may not appear reasonable to perform these examinations routinely following every event of first-trimester miscarriage. 
Therefore, it can be recommended that histopathological examination be performed in selective instances. For instance, when the diagnosis is uncertain preoperatively, when ultrasound suggests a molar pregnancy, when patients are considered of high risk for trophoblastic disease or when inspected the product of conception during ERPC suggests unexpected pathology.

This study seems to be the first local study that evaluated routine histopathological examination after first trimester miscarriage. Further, it was a hospital-based study with a small sample size. However, its findings will escalate the importance of a countrywide study to guide the revision of current practice in this country.

\section{Acknowledgements}

I wish to express my sincere gratitude to Dr. Ibralebbe, Director, Teaching Hospital, Batticaloa, Dr. (Mrs) Partheepan Susithra, and Dr S.Ahilan, Consultant Histopathologists, Dr. Hemika (research assistant) and other staff of Teaching Hospital, Batticaloa for their help during the study period.

\section{References}

1. Hoffman B L, Schorge J L, Karen D et al. First trimester abortion, Williams Gynaecology, McGraw-Hill Education, New York, USA, 3rd edition, 2016:164168

2. Ectopic pregnancy and miscarriage: diagnosis and initial management: NICE guideline Published: 17 April 2019. www.nice.org.uk/guidance/ng126.

Accessed on 10 November 2019

3. Van Look PF, Cottingham J. The World Health Organization's safe abortion guidance document. Am. J. Public Health. 2013;103(4):593-596.

doi:10.2105/AJPH.2012.301204

4. Brook P, Curley M, Sutton B; Brisbane/AU; Evidence-Based Reporting of Suspected Retained Products of Conception Following Miscarriage or Termination of Pregnancy, Poster No.: R0164, Congress: RANZCR-AOCR 2012 Type: Scientific Exhibit. doi:10.1594/ranzcraocr2012/R-016

5. Heath V, Chadwick V, Cooke I, et al. Should tissue from pregnancy termination and uterine evacuation routinely be examined histologically? $\mathrm{Br} \quad \mathrm{J}$ Obstet Gynaecol,2000;107:727-730. doi:10.1111/j.1471-0528.2000.tb13332.x

6. Fram K.M. Histological Analysis of the Products of Conception Following First Trimester Abortion at Jordan University Hospital. Eur J Obstet Gynecol Reprod Biol.2002;105(2):147-149. http://dx.doi.org/10.1016/S03012115(02)00155-0

7. Kirk E, Papageorghiou A T, Condous G, et al, The accuracy of first trimester ultrasound in the diagnosis of hydatidiform mole, Ultrasound Obstet Gynecol, 2007;29:70-75. doi:10.1002/uog.3875

8. Sebire N J, Rees H, Paradinas F et al. The diagnostic implications of routine ultrasound examination in histologically confirmed early molar pregnancies, Ultrasound Obstet Gynecol, 2001;18:662665 https://doi.org/10.1046/j.09607692.2001.00589.x

9. Sharifa Ali Alsibiani. Value of Histopathologic Examination of Uterine Products after First-Trimester Miscarriage. Biomed Research International. 2014;(6):863482. doi:10.1155/2014/863482.

10. Olivier M, Roberts D J, Sengupta E S et al. Prevalence and Factors Associated with Hydatidiform Mole among Patients Undergoing Uterine Evacuation at Mbarara Regional Referral Hospital, Int J Gynaecol Obstet,2018;2018:9561413. doi:10.1155/2018/9561413. 